\title{
Idade relativa em jogadores de futebol profissional do Brasil
}

http://dx.doi.org/10.11606/1807-5509201800040581

\author{
Marlos Adroaldo HEINRICH**** \\ Eder GONÇALVES ${ }^{* *}$ \\ Adeilton Santos GONZAGA* \\ Israel Teoldo COSTA*
}

*Universidade
Federal de Viçosa,
Departamento de
Educação Física,
Viçosa, MG, Brasil.
**Faculdade de
Ciências do Desporto
e Educação Física,
Universidade de
Coimbra, Coimbra,
CO, Portugal.

\section{Resumo}

Durante o processo de formação os jogadores de Futebol são agrupados por categorias etárias de acordo com o seu ano de nascimento. Os jogadores nascidos nos primeiros meses do ano normalmente apresentam uma maturação biológica mais avançada, fato que lhes proporcionam uma vantagem no desempenho da prestação esportiva. Essa vantagem é denominada na literatura esportiva como o efeito da idade relativa. Com isso, o objetivo deste estudo é verificar a existência do efeito da idade relativa entre os jogadores de Futebol no Campeonato Brasileiro Série A. Foram analisados os dados referentes à data de nascimento de 1045 jogadores de Futebol participantes do Campeonato Brasileiro da Série A em 2012. Os dados foram agrupados em quartis, de acordo com o mês de nascimento dos participantes, sendo: $1^{\circ}$ quartil - janeiro, fevereiro e março; $2^{\circ}$ quartil - abril, maio e junho; $3^{\circ}$ quartil - julho, agosto e setembro; $4^{\circ}$ quartil - outubro, novembro e dezembro. Foi utilizada estatística descritiva (frequência e percentual) e para comparação da frequência de jogadores por quartil foi utilizado o teste qui-quadrado $\left(x^{2}\right)$. A análise mostrou a seguinte distribuição dos dados nos quartis: 01 ( $n=326), 02(n=296), 03(n=235)$ e $04(n=188)$. 0 teste qui-quadrado revelou diferenças estatisticamente significativas entre os quartis $\left(x^{2}(3)=43,846\right)$. Com os resultados apresentados, pode-se concluir que o efeito da idade relativa está presente entre os jogadores do Campeonato Brasileiro Série A.

Palavras-Chave: Futebol; Efeito da Idade Relativa; Campeonato Brasileiro.

\section{Introdução}

No Futebol, para se atingir o alto nível competitivo, os jogadores devem apresentar vários fatores que podem ser determinantes para o seu desenvolvimento, como características antropométricas, físicas, sociais, cognitivas, emocionais, táticas e técnicas ${ }^{1,2}$. Tais características podem influenciar de forma positiva ou negativa o desenvolvimento do jogador durante o processo de formação ${ }^{3-6}$.

Portanto, o conhecimento dos fatores que influenciam o desempenho e a consequente seleção dos jogadores pode contribuir de modo significativo para a melhoria do processo de formação desses jogadores de Futebol. Entre as limitaçôes observadas na identificação de talentos, é evidente que durante a puberdade existe a interferência do ritmo de desenvolvimento biológico na capacidade de desempenho do jovem ${ }^{7}$.
Em modalidades esportivas como o Futebol, a inclusão de jovens do sexo masculino em equipes de treinamento pode estar relacionada com a maturação física precoce, na qual a influencia da estatura e da quantidade de massa muscular que os meninos apresentam durante a adolescência pode facilitar o desempenho ${ }^{7}$. Correlacionando com o desempenho tático dos atletas, em um estudo recente, verificaram-se associaçóes positivas entre a eficiência do comportamento tático e o desempenho tático para os princípios Espaço, Penetração e Contenção, sendo que, também foram encontradas associaçóes positivas entre o período do ano de nascimento e o desempenho tático para os jogadores nascidos no $2^{\circ}$ e $3^{\circ}$ quartil, concluindo que, a eficiência do comportamento tático e a data de nascimento influenciaram o 
desempenho tático ${ }^{8}$. Resultados encontrados em outro estudo, também mostraram associaçóes positivas entre a eficiência do comportamento tático e o desempenho tático dos jogadores, além de verificar associação positiva entre a data de nascimento e o Índice de Performance Tática Defensiva dos jogadores?

Contudo, o ano de nascimento é utilizado como critério para o agrupamento das categorias competitivas ao longo do processo de formação de jogadores, como uma forma de diminuir as diferenças resultantes da idade e possibilitar competiçóes mais justas e oportunidades iguais de sucesso para todos ${ }^{10}$. No entanto, é possível observar entre jogadores da mesma categoria etária, diferenças físicas, motoras e psicológicas que resultam em vantagens no desempenho em favor daqueles nascidos nos primeiros meses do ano. Isso se dá em virtude dos jogadores com maior idade cronológica apresentarem estágios mais avançados de maturação biológica ${ }^{11-13}$.

Os diversos estudos em diferentes modalidades que se propuseram a avaliar a categorização das datas de nascimentos dos atletas puderam concluir que, no geral, a distribuição dessas datas de nascimento não é homogênea ${ }^{4,14-16}$. Essas pesquisas concluíram que aqueles atletas que nasceram mais próximo ao início do ano de seleção (primeiro e segundo quartis) apresentam uma super-representação de atletas profissionais, enquanto aqueles atletas nascidos posteriormente (terceiro e quarto quartis) apresentam uma subrepresentação.

O efeito da idade relativa ocorre pela diferença no desenvolvimento dos aspectos físicos, emocionais e intelectuais entre as crianças do mesmo grupo etário ${ }^{17}$. Assim, os jovens do sexo masculino com maior idade cronológica podem apresentar vantagens nas características antropométricas (como estatura, peso corporal, composição corporal), nas capacidades condicionais (como força, velocidade, resistência), no conhecimento cognitivo (como o conhecimento do contexto do jogo e a tomada de decisão) e na capacidade psicológica (como motivação, autoconfiança e autoconceito). Em estudo recente, também foram encontradas relaçóes entre a data de nascimento e o desempenho tático de jogadores de futebol das categorias de base. Foram observadas associaçóes positivas entre o desempenho tático defensivo e o nascimento nos primeiros meses do ano. Isto é um indicativo de que o EIR influencia não só nas capacidades físicas e cognitivas dos jogadores, mas também nas capacidades táticas. Segundo este mesmo estudo, encontrou-se relaçóes positivas entre a eficiência do comportamento e o desempenho tático de jogadores de futebol. Portanto, o treinamento a partir dos princípios táticos, que podem modular o comportamento no jogo, parece ser pertinente para o desenvolvimento do atleta, uma vez que esta é uma variável que influencia diretamente no desempenho do mesmo ${ }^{1}$.

Desse modo, principalmente em situações competitivas, os jovens mais velhos tendem a ter um desempenho superior e, como consequência, possuem maiores oportunidades de acesso ao treinamento. Em contrapartida, aqueles que náo se destacam, muitas vezes por fatores maturacionais, acabam sendo excluídos precocemente do processo de treinamento ${ }^{18}$. Esta tendência na seleçáo de jogadores que apresentam um desenvolvimento físico precoce, em detrimento daqueles com desenvolvimento esperado ou tardio, pode ser um erro, pois, não necessariamente, os indivíduos precoces continuarão apresentando essa vantagem na idade adulta ${ }^{19}$.

Até o momento do estudo, notou-se uma carência nos estudos científicos sobre o efeito da idade relativa que envolve atletas brasileiros de futebol profissional. É verificado um número maior de publicaçóes na literatura internacional, originando uma dificuldade na comparação de dados considerando diferenças no calendário esportivo competitivo, e também publicaçôes nas categorias de formaçáo, onde são encontrados os resultados com maior efeito da idade relativa. Considerando os fatos, duas hipóteses foram destacadas: a distribuição das frequências de datas de nascimento de atletas pertencentes à equipes brasileiras de futebol é semelhante a distribuição de frequências das equipes internacionais, e não há diferença entre a distribuição de frequência de datas de nascimento entre as categorias de base e categoria profissional no futebol brasileiro. Neste sentido, é importante o conhecimento do efeito da idade relativa em jogadores no Futebol brasileiro. Sendo assim, o objetivo deste estudo foi analisar a existência do efeito da idade relativa entre os jogadores de Futebol que disputaram o Campeonato Brasileiro Série A. 


\section{Materiais e Método}

\section{Amostra}

A amostra deste estudo foi constituída pelos dados referentes à data de nascimento de 1045 jogadores de Futebol que participaram do Campeonato Brasileiro Série A no ano de 2012.

\section{Procedimento de coleta de dados}

Os dados foram coletados através das informações referentes à data de nascimento dos jogadores, disponíveis no site http://www.ogol.com.br. Este site apresenta dados de clubes, jogadores, seleçóes, ligas e competiçóes de todo o mundo. Os dados coletados foram inseridos numa planilha do Windows Excel ${ }^{\circ}$, onde foram agrupados em quartis, de acordo com o mês de nascimento dos jogadores, sendo: $1^{\circ}$ quartil (Q1) - janeiro, fevereiro e março; $2^{\circ}$ quartil (Q2) - abril, maio e junho; $3^{\circ}$ quartil (Q3) - julho, agosto e setembro; $4^{\circ}$ quartil (Q4) - outubro, novembro e dezembro.

\section{Análise estatística}

Foram realizadas as análises descritivas de frequência e percentual para se obter informaçóes sobre diferentes aspectos da amostra. O teste Qui-quadrado $\left(\mathrm{x}^{2}\right)$ foi utilizado para comparar a frequência de jogadores nascidos nos diferentes quartis. Também, foram calculados o standard residual (std residual $=\mathrm{O}-\mathrm{E} / \sqrt{\mathrm{E}}$ ), para determinar qual quartil contribui para o valor do $\mathrm{x}^{2}$, e o effect size $\left(\mathrm{w}=\sqrt{ } \mathrm{x}^{2} / \mathrm{n}\right)$.

Todos os procedimentos estatísticos utilizaram o nível de significância de $p<0,05$ e foram realizados no software SPSS (Statistical Package for Social Science) for Windows ${ }^{\oplus}$, versão 20.0.

\section{Resultados}

A TABELA 1 apresenta os resultados da distribuição dos jogadores por seu quartil de nascimento e os valores do qui-quadrado $\left(\mathrm{x}^{2}\right)$. $\mathrm{O}$ teste qui-quadrado $\left(\mathrm{x}^{2}\right)$ revelou diferenças estatisticamente significativas na distribuição dos jogadores. Os valores do standard residual revelaram maiores discrepâncias no Q1 $(S R=4,00)$ e Q4 (SR = -4,53).

TABELA 1 - Distribuição dos jogadores pelo quartil de nascimento

\begin{tabular}{lccc}
\hline Quartis & Frequência & Percentual & Standard Residual \\
\hline Q1 & 326 & 31,2 & 4,00 \\
Q2 & 296 & 28,3 & 2,15 \\
Q3 & 235 & 22,5 & $-1,62$ \\
Q4 & 188 & 18,0 & $-4,53$ \\
\hline
\end{tabular}

$x^{2}=43,846$; $p<0,001 ; w($ effect size) $=0,2$.

\section{Discussão}

O objetivo deste estudo foi analisar a existência do efeito da idade relativa entre os jogadores de Futebol que disputaram o Campeonato Brasileiro Série A. Os resultados da distribuição dos jogadores pelos quartis de nascimento revelaram uma maior frequência de participantes nascidos nos primeiros quartis. Estes resultados caracterizam a presença do efeito da idade relativa, que favorece a participação dos jogadores nascidos nos primeiros meses, em detrimento àqueles que nasceram nos últimos meses do ano, em competiçôes esportivas. Os dados analisados apresentaram uma distribuição desigual dos meses de nascimento, com uma maior frequência de nascidos no primeiro quartil e no segundo quartil.

Em outro estudo semelhante, também foi encontrado esta diferença para a amostra avaliada, houve uma tendência dos jogadores do futebol brasileiro nascidos no início do ano, mais especificamente entre janeiro e março, ainda, de 
acordo com os resultados obtidos, identificouse uma semelhança na distribuiçáo de datas de nascimento com os dados estatísticos da população brasileira, que correspondeu a 28,3\% para Q1 e $28,1 \%$ para Q2. Considerando o restrito acesso aos dados da população brasileira, houve a impossibilidade de fazer análise estatística para comparação entre os quartis, embora visualmente note-se uma tendência de nascimentos no início do ano referente a 1984-2002. Dessa maneira, parece náo consistir na forma como os clubes brasileiros fazem o processo de detecção e seleção de talentos, preferem os mais velhos e maturados, mas, sim uma tendência de nascimentos nos primeiros meses do ano da populaçáo brasileira de uma maneira geral. Entretanto, é uma situaçáo que deve ser analisada com cautela considerando a exclusão automática de possíveis jovens talentos por parte de treinadores justificada apenas por uma tendência populacional, descarta-se a possibilidade do desenvolvimento de uma carreira esportiva para esses jovens ${ }^{20}$.

A maior participação dos jogadores nascidos nos meses iniciais de agrupamento etário pode ser uma consequência da seleçáo dos jogadores mais maturados, dentro de determinada categoria, durante a infância e início da adolescência. Ao final da adolescência ou na fase adulta, não existe mais interferência direta no desempenho relacionado com as vantagens associadas à idade relativa, ou ao ritmo de maturação biológica ${ }^{18}$. Porém, é comum observar uma maior frequência de jogadores nascidos nos primeiros meses do ano compondo a categoria profissional, pois aproveitaram as vantagens proporcionadas por esses fatores em idades anteriores.

Da forma como são organizadas as categorias de base, no processo de formação esportiva, os jovens nascidos nos primeiros meses do ano possuem maiores chances de serem selecionados pelas equipes, pois se encontram em estágios mais avançados de maturação biológica. Nos períodos pré-púberes e púberes, existe uma tendência dos garotos mais velhos apresentarem características antropométricas e capacidades condicionais (força, velocidade e resistência) mais desenvolvidas, proporcionando, aos mesmos, maiores chances de serem selecionados para participar do processo de treinamento nas equipes ${ }^{13,21}$. Isso pode representar essa superioridade de jogadores nascidos nos primeiros meses do ano nas equipes de Futebol na categoria profissional.
A presença do efeito da idade relativa é mais evidente nos esportes com mais contato físico, como o Futebol, no qual os atletas necessitam de uma elevada capacidade de velocidade, bem como, agilidade dos movimentos, além de um excelente domínio espaço temporal. Porém, essas vantagens são transitórias, uma vez que são influenciadas pela idade cronológica e desenvolvimento biológico, e não necessariamente continuarão após o fim da adolescência ${ }^{22}$. Além disso, o efeito da idade relativa tem sido influenciado por outros fatores como o nível de competitividade da modalidade, o gênero e a posição dos jogadores, entre outros ${ }^{23}$.

Cabe ressaltar, que apesar de não ter sido avaliada no presente estudo, deve-se destacar o papel da maturação biológica e a sua influência no que diz respeito aos efeitos da idade relativa ${ }^{24}$. $\mathrm{Na}$ maioria das vezes, a identificação e a seleção de talentos ocorrem em um momento de instabilidade das variáveis que influenciam no desempenho, ou seja, em um período em que as diferentes velocidades de maturação biológica interferem ${ }^{22}$. A exclusão ou desistência de indivíduos nascidos nos últimos meses do ano, durante os primeiros anos de prática no Futebol, pode ter influenciado o processo de formaçáo esportiva dos jogadores e, consequentemente, a composição das equipes analisadas no presente estudo. Jogadores que mostram um estado avançado em seu processo maturacional com diferenças óbvias em habilidades funcionais têm vantagens, dado que recebem mais oportunidades de treinamento e competição ${ }^{25,26}$. Por exemplo, a maturação apresenta uma relação com o desenvolvimento da percepçáo periférica, e essa tem relação com a eficiência do comportamento tático. Nesse sentido, vale ressaltar a importância de considerar a importância da análise da maturação em estudos com jovens jogadores de futebol ${ }^{27}$.

Outro fator que pode ser levado em consideração, e que pode ter influência no resultado da distribuição dos jogadores analisados nesse estudo, é que os jovens com maior idade cronológica podem também levar maiores vantagens no aspecto cognitivo, ou seja, o maior tempo de experiência pode proporcionar um maior conhecimento do contexto do jogo, aumentando as chances de maior precisão e velocidade nas decisóes acerca das açóes motoras e elaboração de estratégias ${ }^{17}$.

A seleção de jogadores priorizando os aspectos técnico-táticos, ao invés dos atributos físicos, é uma das estratégias sugeridas para minimizar as discrepâncias da distribuição dos meses de 
nascimento no Futebol ${ }^{13}$. Porém deve-se considerar que a experiência acumulada relacionada com a idade continuaria a influenciar nestes aspectos ${ }^{28}$. Deve-se pensar em soluções para evitar a exclusão precoce no Futebol, a criação de outros níveis de torneios (séries ouro, prata e bronze para uma mesma categoria), por exemplo, poderia motivar os jovens, principalmente nos anos iniciais de aprendizagem para a prática do Futebol.

Referindo-se ao desempenho tático, em estudos já realizados com jogadores de futebol da categoria sub-13, os nascidos nos primeiros meses do ano náo apresentaram vantagens em relação aos nascidos em outros períodos. Dessa forma, torna-se plausível concluir que o efeito da idade relativa (EIR) não se apresentou como um fator influente no desempenho tático dos jogadores de futebol da categoria sub- $13^{24}$.

É necessário que os treinadores utilizem cada vez mais de ferramentas científicas durante o processo de formação esportiva e que haja uma conscientização sobre a existência do efeito da idade relativa e que ocorra uma mudança na filosofia de trabalho, estabelecendo um balanço entre o sucesso de curto prazo e o desenvolvimento em longo prazo ${ }^{10}$. Ressaltase, também, a necessidade da elaboraçáo de programas no Futebol, onde os excluídos (possivelmente nascidos nos últimos meses) possam continuar a prática esportiva em outros níveis e assim tenham a chance de retornar ao alto rendimento.
Com os resultados apresentados, pode-se concluir que o Efeito da Idade Relativa está presente nos jogadores do Campeonato Brasileiro Série A de 2012, onde, observa-se uma maior frequência de jogadores nascidos nos primeiros meses do ano. Mudanças periódicas na data de início do ano etário para o Futebol, a conscientização dos treinadores para enfatizarem menos os aspectos físicos, e a formação das categorias ano a ano, diminuindo este intervalo existente de dois anos, poderiam minimizar os efeitos da idade relativa entre os jogadores de Futebol.

Em se tratando de futebol, onde fatores cronológicos e biológicos podem gerar vantagens ou desvantagens, sugerem-se novos estudos em que haja o acompanhamento de jogadores em formação, considerando atributos físicos, maturacionais e técnicos relacionados ao desempenho no ambiente real de jogo, levando em consideração outras variáveis como a tomada de decisão, ou até mesmo do desempenho tático desses jogadores. Podendo, ainda, a realização de estudos que quantifiquem o número de jovens jogadores, identificados como talentos na fase de formação, que se tornaram atletas profissionais em equipes de alto nível competitivo. Novos estudos podem dar o suporte para melhorar no processo de seleção de atletas, e no entendimento do efeito da idade relativa.

\begin{abstract}
Relative age in professional Football players of Brazil

Throughout the development process, players are grouped by age levels, according to their year of birth, whereas only their chronological age is considered, rather than their biological maturation. Athletes born in the first months of the year typically have a more advanced biological maturation, a fact that gives them an advantage in performance during training and games, a phenomenon referred to by the sports literature as the relative age effect. The objective of this study is to verify the existence of the relative age effect among soccer players in the Brazilian Serie A Championship. The data regarding the date of birth of 1045 football players participating in the Brazilian Serie A Championship in 2012 were analyzed. Data were grouped into quarters according to month of birth of the participants, as follows: 1st quarter - January, February and March; 2nd quarter - April, May and June; 3rd quarter - July, August and September; 4th quarter - October, November and December. The analysis showed the following distribution of the data in quarters: $01(n=326), 02(n=296), 03(n=235)$ and $04(n=188)$. With the results presented, it can be concluded that the Relative Age Effect is present in players in the Brazilian Serie A Championship, since there is a higher frequency of players born in the first quartile.
\end{abstract}

KEYWords: Football; Relative Age Effect; Campeonato Brasileiro. 


\section{Conflito de interesses}

Tive acesso irrestrito a todos os dados deste estudo e assumo inteira responsabilidade pela integridade dos dados e pela precisão da análise deles. Não houve, portanto, conflitos de interesse na realização desse estudo.

\section{Agradecimentos}

Este trabalho teve o apoio da Secretaria de Estado de Turismo e Esportes (Setes - MG) através da Lei Estadual de Incentivo ao Esporte, da FAPEMIG, da CAPES, do CNPQ, da FUNARBE, da Reitoria, Pró-Reitoria de Pesquisa e Pós-Graduação e do Centro de Ciências Biológicas e da Saúde da Universidade Federal de Viçosa.

\section{Referências}

1. Costa IT, Garganta J, Greco PJ, Mesquita I, Seabra A. Influence of relative age effects and quality of tactical behaviour in the performance of youth soccer players. Int J Perform Anal Sport. 2010;10(2):82-97.

2. Moraes LCCA, Penna EM, Ferreira RM, Costa VT, Matos AF. Análise do quartil de nascimento de atletas profissionais de futebol. Pensar Prát. 2009;12(3):1-9.

3. Berthon P, Fellman N. General review of maximal aerobic velocity measurement al laboratory. J Sports Med Phys Fitness. 2002;42(3):257-66.

4. Davids K, Lees A, Burwitz L. Understanding and measuring coordination and control in kicking skills in soccer: Implications for talent identification and skill acquisition. J Sports Sci. 2000;18(9):703-14.

5. Kemi O, Hoff J, Engen L, Helgerud J, Wisløff U. Soccer specific testing of maximal oxygen uptake. J Sports Med Phys Fitness. 2003;43(2):139-44.

6. Reilly T, Bangsbo J, Franks A. Anthropometric and physiological predispositions for elite soccer. J Sports Sci. 2000;18(9):669-83.

7. Bojikian LP, Teixeira CP, Böhme MTS, Ré AHN. Relaçôes entre crescimento, desempenho motor, maturação biológica e idade cronológica em jovens do sexo masculino. Rev Bras Educ Fís Esporte. 2005;19(2):153-62.

8. Machado GF, Scaglia AJ, Costa IT. Influência do efeito da idade relativa e do comportamento tático sobre o desempenho tático de jogadores de futebol da categoria Sub-17. Rev Educ Fís UEM. 2015;26(2):223-31.

9. Andrade MOC, Costa IT. Como a eficiência do comportamento tático e a data de nascimento condicionam o desempenho de jogadores de futebol? Rev Bras Educ Fís Esporte. 2015;29(3):465-73.

10. Helsen WF, Van Winckel J, Williams AM. The relative age effect in youth soccer across Europe. J Sports Sci. 2005;23(6):629-36.

11. Carling C, Le Gall F, Reilly T, Williams A. Do anthropometric and fitness characteristics vary according to birth date distribution in elite youth academy soccer players? Scand J Med Sci Sports. 2009;19(1):3-9.

12. Wattie N, Cobley S, Baker J. Towards a unified understanding of relative age effects. J Sports Sci. 2008;26(13):1403-9.

13. Folgado HA, Caixinha PF, Sampaio J, Maçâs V. Efeito da idade cronológica na distribuição dos futebolistas por escalōes de formação e pelas diferentes posiçóes especificas. Rev Port Ciên Desp. 2006;6(3):349-55.

14. Costa VT, Simim MA, Noce F, Costa IT, Samulski DM, Moraes LC. Comparison of relative age of elite athletes participating in the 2008 Brazilian soccer championship series A and B. Motricidade. 2009;5(3):13-7.

15. Delorme N, Boiché J, Raspaud M. Relative age effect in elite sports: Methodological bias or real discrimination? Eur J Sport Sci. 2010;10(2):91-6.

16. Penna EM, Moraes L. Efeito relativo da idade em atletas brasileiros de futsal de alto nível. Motriz. 2010;16(3):658-63.

17. Musch J, Grondin S. Unequal competition as an impediment to personal development: A review of the relative age effect in sport. Dev Rev. 2001;21(2):147-67.

18. Carli GC, Luguetti CN, Ré AHN, Böhme MTS. Efeito da idade relativa no futebol. Rev Bras Ciênc Mov. 2009;17(3):25-31.

19. Böhme MTS. Treinamento a longo prazo e o processo de deteç̧ão, seleção e promoção de talentos esportivos. Rev Bras Ciênc Esporte. 2000;21(2/3). 
20. Rabelo FN, Pasquarelli BN, Matzenbacher F, et al. Efeito da idade relativa nas categorias do futebol brasileiro: critérios de seleção ou uma tendência populacional? Rev Bras Ciênc Esporte. 2016;38(4):370-5.

21. Vincent J, Glamser FD. Gender differences in the relative age effect among US Olympic Development Program youth soccer players. J Sports Sci. 2006;24(4):405-13.

22. Martindale RJ, Collins D, Daubney J. Talent development: a guide for practice and research within sport. Quest. 2005;57(4):353-75.

23. Schorer J, Cobley S, Büsch D, Bräutigam H, Baker J. Influences of competition level, gender, player nationality, career stage and playing position on relative age effects. Scand J Med Sci Sports. 2009;19(5):720-30.

24. Silva T, Garganta J, Brito J, Cardoso F, Teoldo I. Influência do efeito da idade relativa sobre o desempenho tático de jogadores de futebol da categoria sub-13. Rev Bras Ciênc Esporte. 2018;40(1):54-61.

25. Figueiredo AJ, Gonçalves CE, Coelho e Silva MJ, Malina RM. Youth soccer players, 11-14 years: maturity, size, function, skill and goal orientation. Ann Hum Biol. 2009;36(1):60-73.

26. Malina RM, Peña Reyes ME, Figueiredo AJ, et al. Skeletal age in youth soccer players: implication for age verification. Clin J Sport Med. 2010;20(6):469-74.

27. Gonçalves E, Noce F, Barbosa MAM, Figueiredo AJ, Hackfort D, Teoldo I. Correlation of the peripheral perception with the maturation and the effect of the peripheral perception on the tactical behaviour of soccer players. Int J Sport Exerc Psychol [Internet]. 2017 [citado 22 jul 2019]. Disponível em: https://doi.org/10.1080/1612197X.2017.1329222.

28. Simmons C, Paull GC. Season-of-birth bias in association football. J Sports Sci. 2001;19(9):677-86.

\begin{tabular}{r|r} 
ENDEREço & \\
Marlos Adroaldo Heinrich & Recebido para publicação: 06/08/2015 \\
Avenida Conímbriga, Pavilhão 3 & Revisão: 15/11/2017 \\
3040-248 - Coimbra - PORTUGAL & Aceito: 20/12/2017 \\
e-mail: marlosmedianeira@hotmail.com; & \\
marlosmedianeirapr@gmail.com &
\end{tabular}

